\title{
Joint reconstruction of activity and attenuation in dynamic PET
}

\author{
Alaleh Rashidnasab, Member, IEEE, Alexandre Bousse, Beverley F Holman, Member, IEEE, \\ Brian F Hutton, Senior Member, IEEE, and Kris Thielemans Senior Member, IEEE
}

\begin{abstract}
Joint reconstruction of attenuation and emission in positron emission tomography (PET) using the maximum likelihood activity and attenuation estimation (MLAA) algorithm was proposed in the past. However, cross-talk between the activity and attenuation estimation limits the usefulness of MLAA for PET data without time-of-flight (TOF) information. This work introduces dynamic MLAA (dMLAA), an extension of the MLAA algorithm for dynamic data, to jointly reconstruct the activity distributions and a single attenuation map. The hypothesis is that using information from multiple dynamic emission frames may improve the estimated attenuation map compared to using static PET data. Preliminary results using dMLAA algorithm showed that use of multiple dynamic emission frames slightly improves the reconstructed attenuation map (especially in bones, cavities and lesion area) compared to using a single emission frame. However, without TOF, the reconstructed map still suffers from ill-posedness of the problem despite the additional dynamic information. The reconstruction may be improved for tracers that present a higher inter- and intra- dynamic frame contrast and edge variability.
\end{abstract}

Index Terms-dynamic PET, MLAA, joint activity and attenuation estimation, dMLAA.

\section{INTRODUCTION}

A TTENUATION correction is a key step in quantitative reconstruction of a tracer distribution in PET. While transmission scans can be acquired [1], an alternative approach is to estimate the attenuation map from the emission data. Previously, a reconstruction method based on joint reconstruction of attenuation and emission PET imaging, known as maximum likelihood activity and attenuation estimation (MLAA), was proposed [2]. The extension of the MLAA reconstruction algorithm for time-of-flight (TOF) PET imaging showed promising results where the so-called "cross-talk" effect was eliminated and the attenuation sinogram determined up to a constant [3], [4]. This work introduces dynamic MLAA (dMLAA), an extension of the MLAA algorithm for dynamic PET data, to jointly reconstruct the activity distributions and a single attenuation map. The hypothesis is that using information from multiple dynamic emission frames improves the estimated single attenuation map compared to using static PET data.

This work was supported by UK EPSRC (EP/K005278/1) and the NIHRfunded UCLH Biomedical Research Centre.

A. Rashidnasab, A. Bousse, B. F. Holman, B. F. Hutton and K. Thielemans are with the Institute of Nuclear Medicine, University College London, London, UK (email: a.rashidnasab@ucl.ac.uk).

B. F. Hutton is also with the Centre for Medical Radiation Physics, University of Wollongong, Wollongong, Australia.

Manuscript received November 30, 2016.

\section{Methods}

\section{A. Algorithm description}

dMLAA consists of two algorithms with an alternating update. In each iteration of dMLAA, the activity distribution for each frame is first updated with maximum likelihood expectation maximisation (MLEM) using the current attenuation map estimate, Eq.(1). Second, the attenuation map is updated with maximum likelihood for transmission tomography (MLTR) using the integrated activity distributions estimated in the first step, Eq.(3).

$$
\begin{gathered}
\boldsymbol{f}_{m}^{(i+1)}=\frac{\boldsymbol{f}_{m}^{(i)}}{\boldsymbol{P}\left(\boldsymbol{\mu}^{(i)}\right)^{\top} \mathbf{1}} \boldsymbol{P}\left(\boldsymbol{\mu}^{(i)}\right)^{\top} \frac{\boldsymbol{g}_{m}}{\boldsymbol{P}\left(\boldsymbol{\mu}^{(i)}\right) \boldsymbol{f}_{m}^{(i)}}, \\
\boldsymbol{P}\left(\boldsymbol{\mu}^{(i)}\right)=\operatorname{diag}\left\{\mathrm{e}^{-\boldsymbol{P} \boldsymbol{\mu}^{(i)}}\right\} \boldsymbol{P}, \\
\boldsymbol{\mu}^{(i+1)}=\boldsymbol{\mu}^{(i)}+\frac{\boldsymbol{P}^{\top}\left(\sum_{m=1}^{M}\left(\overline{\boldsymbol{g}}\left(\boldsymbol{f}_{m}^{(i+1)}, \boldsymbol{\mu}^{(i)}\right)-\boldsymbol{g}_{m}\right)\right)}{\boldsymbol{P}^{\top}\left(\sum_{m=1}^{M}\left(\overline{\boldsymbol{g}}\left(\boldsymbol{f}_{m}^{(i+1)}, \boldsymbol{\mu}^{(i)}\right) \cdot \boldsymbol{P} \mathbf{1}\right)\right)}
\end{gathered}
$$

where $\boldsymbol{f}_{m}, m=1,2, \ldots, M$, is the $m^{\text {th }}$ reconstructed dynamic frame, $\boldsymbol{g}_{m}$ are the corresponding measured counts, $\overline{\boldsymbol{g}}\left(\boldsymbol{f}_{m}, \boldsymbol{\mu}\right)=\boldsymbol{P}(\boldsymbol{\mu}) \boldsymbol{f}_{m}$ are the expected counts and $\boldsymbol{\mu}$ is the estimated attenuation map. dMLAA can be extended to Maximum A Posteriori (MAP) as in [2].

\section{B. Evaluation}

Having a higher inter- and intra- dynamic frame contrast and edge variability may improve the reconstructed attenuation map when using the dMLAA algorithm. To demonstrate this a checkerboard phantom was created (Fig. 2(a)) to simulate 20 dynamic emission frames where the white squares on the left half had a linear time-activity-curve (TAC) increasing from 10 to 200 across the frames as shown in Fig. 1. The white squares on the right half on the phantom had opposite time behaviour. All the black squares had a constant activity. The true attenuation map consisted of water with two sub-regions with the attenuation of lung, see Fig. 2(d).

To investigate the performance of dMLAA in a more realistic setting, the algorithm was further tested on two phantoms created from reconstructed clinical images of dynamic FDG PET/CT data of brain and thorax scans. The acquisition durations were $50 \mathrm{~min}$ and $20 \mathrm{~min}$ for the brain and thorax respectively. To add an extra feature, a lesion (cube) with an FDG TAC representative of an avid lesion was inserted into the created thorax phantom. Sec.III summarises the dMLAA results when using (1) the final dynamic frame, (2) all available 


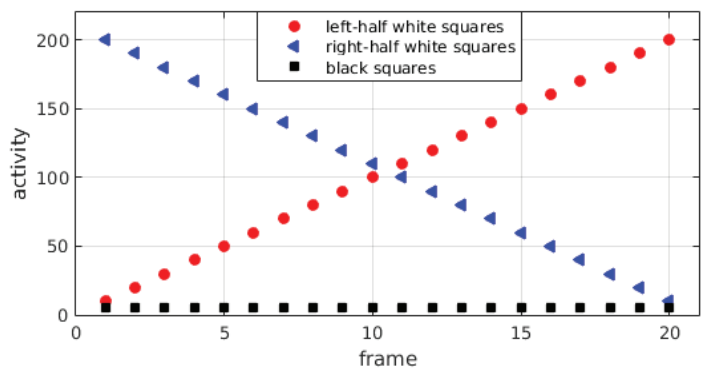

Fig. 1

dynamic frames to reconstruct the attenuation map and the activity distributions.

The emission frames were initialised with uniform non-zero values. The attenuation map was initialised to $80 \%$ of the soft tissue attenuation and the outer surface was constrained to the true attenuation phantom. 250 iterations for MLAA was used in the simulations, with 20 MLEM iterations and 5 MLTR iterations within each MLAA iteration. A parallel beam geometry was used for the projectors. Scatter and random contributions were ignored in this preliminary evaluation.

\section{RESULTS}

Fig. 2 shows the reconstruction results of the checkerboard phantom. As seen in Fig. 2(f) and the attenuation profile in Fig. 2(h), the reconstructed attenuation map from multiple dynamic frames has better reconstruction of the lung subregions and less "cross-talk" compared to reconstruction from one dynamic frame in Fig. 2(e). Performance of dMLAA degraded when increasing the size of the "tiles" in the activity image (results not shown).

Fig. 3 shows the result of dMLAA reconstruction of the brain phantom. Fig. 3(b) and Fig. 3(c) show the reconstructed activity distributions of the final dynamic frame when using one dynamic frame and all dynamic frames respectively. The comparison of the reconstructed attenuation maps to the true attenuation map (Fig. 3(d)) shows that more features (bones and cavities) were reconstructed using multiple dynamic frames (Fig. 3(f)) compared to using one frame (Fig. 3(e)) in the joint reconstruction. However, the attenuation map was not completely recovered due to the ill-posed nature of the problem.

Fig. 4 shows the dMLAA reconstruction of the thorax phantom. Fig. 4(f) shows that using multiple dynamic emission frames for reconstruction of the attenuation map improved the reconstruction of the lesion and the posterior regions of the lungs compared to the attenuation map in Fig. 4(e) reconstructed using one emission frame only.

\section{CONCLUSION}

Use of multiple dynamic emission frames slightly improves the reconstructed attenuation map using the dMLAA algorithm. However, without TOF, the reconstructed map suffers from ill-posedness of the problem despite the additional dynamic information. The reconstruction may be improved for tracers that present high variation of contrast between frames and within each frame.

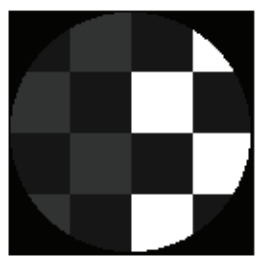

(a)

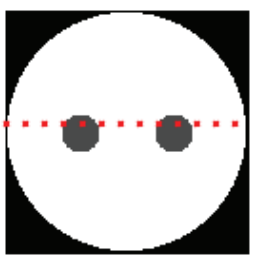

(d)

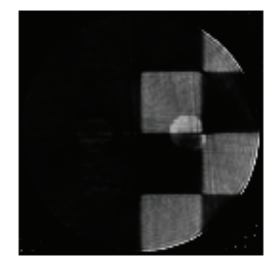

(b)

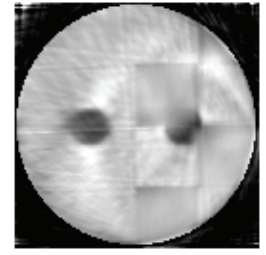

(e)

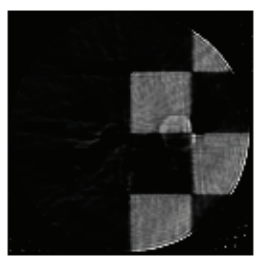

(c)

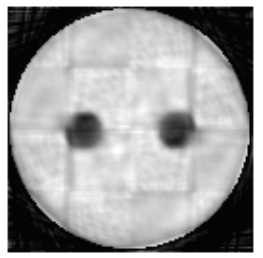

(f)

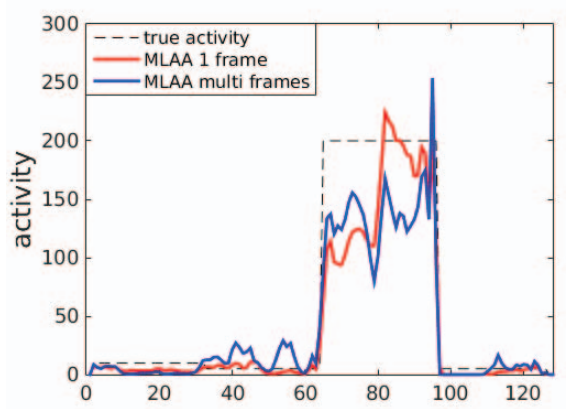

(g)

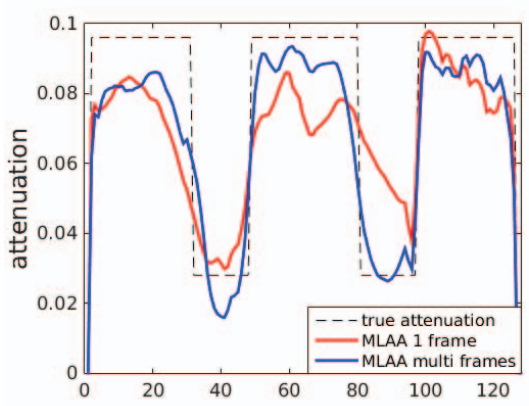

(h)

Fig. 2: dMLAA on the simulated checkerboard phantom. (a) True activity, (b) reconstructed activity using one frame, (c) reconstructed activity using all dynamic frames, (d) true attenuation, (e) reconstructed attenuation using one frame, (f) reconstructed attenuation using all dynamic frames, (g) activity profiles, (h) attenuation profiles. Red dotted line on (d) displays the profile location used in (a)-(f) to produce (g) and (h).

\section{REFERENCES}

[1] P. E. Kinahan, D. W. Townsend, T. Beyer, and D. Sashin, "Attenuation correction for a combined 3-D PET/CT scanner," Med. Phys., vol. 25, no. 10 , pp. 2046-2053, 1998.

[2] J. Nuyts, P. Dupont, S. Stroobants, R. Benninck, L. Mortelmans, and P. Suetens, "Simultaneous maximum A Posteriori reconstruction of attenuation and activity distributions from emission sinograms," IEEE Trans. Med. Imag., vol. 18, no. 5, pp. 393-403, 1999.

[3] A. Rezaei, M. Defrise, G. Bal, C. Michel, M. Conti, C. Watson, and J. Nuyts, "Simultaneous reconstruction of activity and attenuation in timeof-flight PET," IEEE Trans. Med. Imag., vol. 31, no. 12, pp. 2224-2233, 2012.

[4] M. Defrise, A. Rezaei, and J. Nuyts, "Time-of-flight PET data determine the attenuation sinogram up to a constant," Phys. Med. Biol., vol. 57, no. 4, pp. 885-899, 2012. 


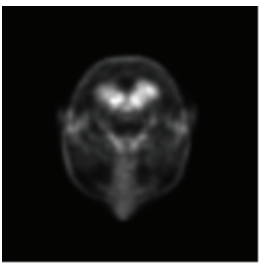

(a)

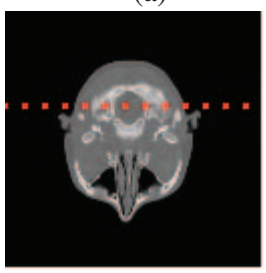

(d)

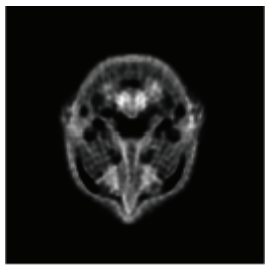

(b)

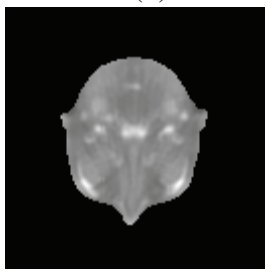

(e)

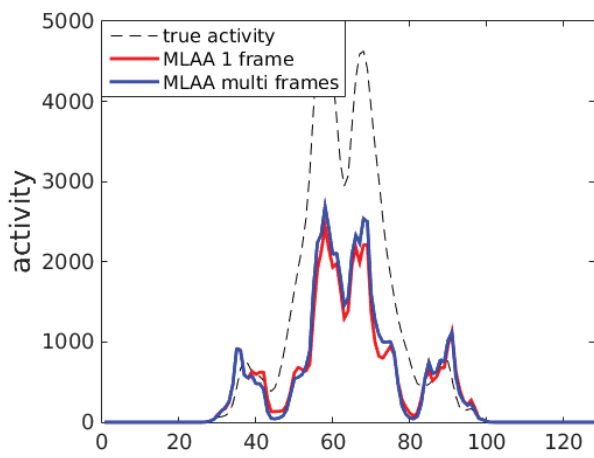

(g)

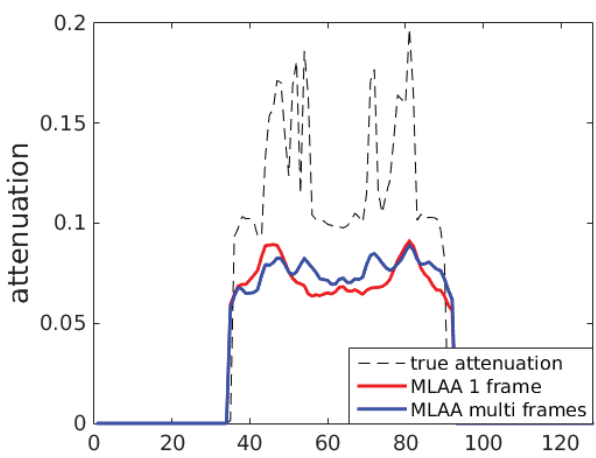

(h)

Fig. 3: dMLAA on the phantom created from reconstructed clinical images of dynamic PET/CT brain data. (a) True activity (frame 18), (b) reconstructed activity (same frame as in a) using one frame, (c) reconstructed activity (same frame as in a) using all dynamic frames, (d) true attenuation, (e) reconstructed attenuation using one frame, (f) reconstructed attenuation using all dynamic frames, (g) activity profiles, (h) attenuation profiles. Red dotted line on (d) displays the profile location used in (a)-(f) to produce (g) and (h).

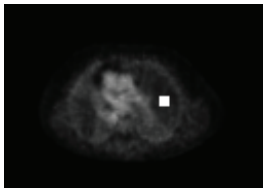

(a)

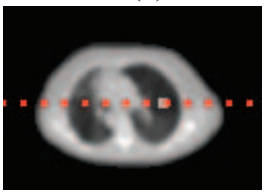

(d)

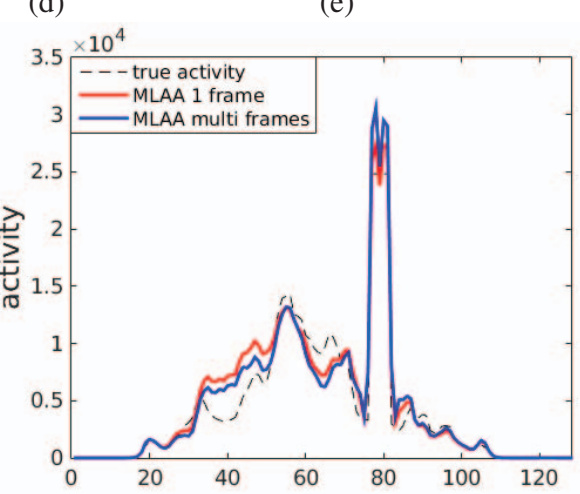

(g)

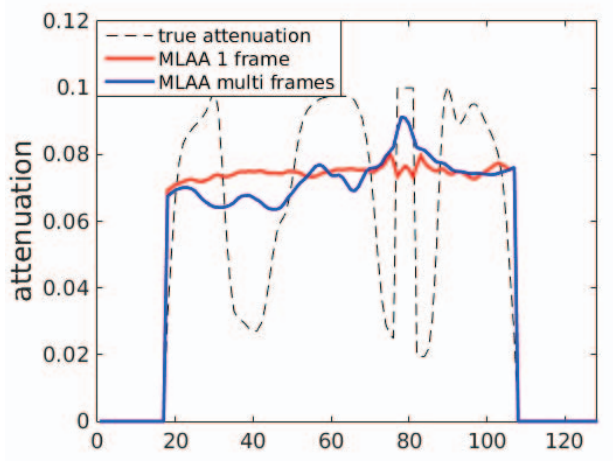

(h)

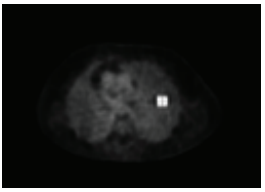

(c)

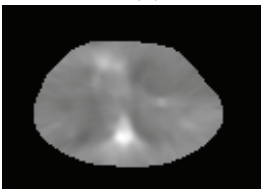

(f)

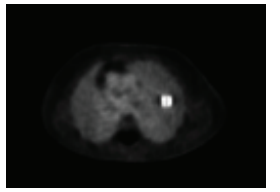

(b)

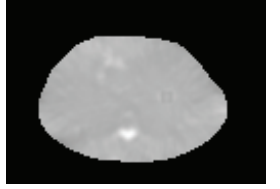

(e) 